\title{
Average Block Error Probability in the Reverse Link of a Packet DS/CDMA System under Rayleigh Fading Channel Conditions
}

\author{
Jordi Pérez-Romero, Luis G. Alonso, and Ramón Agustí
}

\begin{abstract}
Analytic expressions have been derived for the probability density functions of the total signal-to-interference ratio when considering mixed-type interference sources in the reverse link of a DS/CDMA packet radio system with Rayleigh fading. Four different scenarios were studied, and the corresponding expressions are presented for each situation. The users are divided into two groups: those that apply a closed-loop power control and those that only use an open-loop power control. By making use of these expressions, the mean block error probability is derived as a function of the number of total interfering users in each group.
\end{abstract}

Index Terms-Code division multiaccess, packet radio, power control, Rayleigh channels.

\section{INTRODUCTION}

W HEN evaluating the capacity of DS/CDMA systems, great effort has been focused on calculating the average bit error probability in a scenario where all the users generate the same type of interference. However, in a packet transmission scheme it might be possible to find users that are applying different power control schemes. Some of them may only apply an open-loop power control as they are sending their request access packets or even because of the shortness of the message they are to transmit, while others will be able to apply a closed-loop power control as they are continuously transmitting with some reserved resources that include a downlink control channel carrying power control commands.

In a packet transmission system, performance is defined by the average block error probability. Several expressions have been widely used to calculate the average bit error probability in a Rayleigh fading environment [1], [2]. However, these expressions are not directly applicable to the evaluation of the average block error probability, as the statistics of the bit error probability must be taken into account instead of employing only the mean value.

On the basis of this framework, in this letter we present analytical expressions for the probability density functions (pdf's) of the total signal-to-interference ratio in a DS/CDMA environ-

\footnotetext{
Manuscript received June 9, 1999. The associate editor coordinating the review of this letter and approving it for publication was Dr. K. Sriram. This work was supported by CYCIT project TIC 98-684 and by a grant of the Spanish Education Ministry.

The authors are with the Department of Signal Theory and Communications, Polytechnic University of Catalonia (UPC), 08034 Barcelona, Spain (e-mail: jorperez@tsc.upc.es).

Publisher Item Identifier S 1089-7798(00)02938-0.
}

ment when considering different scenarios with mixed-type interference sources. Our contribution focuses on two cases with a mixture of users employing both open and closed-loop power control, by distinguishing the situation in which the desired user works with either one or the other power control technique. The derived pdf's are used to obtain analytical expressions for evaluating the average block error probability when using block channel codes with a given correcting capability.

\section{Average Block Error Probability IN A DS/CDMA ENVIRONMENT}

We consider a DS/CDMA packet transmission network. Users generate and transmit $L$-bit packets. They use pseudo-random sequences with spreading factor $S_{f}$. The channel is time slotted, and each $L$-bit packet is transmitted within a single slot. The channel coherence time [2] is assumed to be greater than the packet transmission time, that is, the response of the channel does not change during a packet transmission. Consequently, the same fading applies to all the bits of a given packet.

Four different scenarios are analyzed below.

\section{A. All Users with Closed-Loop Power Control}

We consider a group of $m+1$ users. Therefore, for a given target user, there are $m$ interferers. Perfect closed-loop power control is considered, so that the received power at the base station is assumed to be the same for each user. For a large number of users, the combined interference coming from the different packets can be approximated by a Gaussian random variable. This is known as the Gaussian approximation. This approximation has been widely used in the literature due to its simplicity and sufficient accuracy, even for a small number of users [3]. We will denote the total signal-to-interference ratio (SIR) as $\gamma$. Under these assumptions, considering BPSK modulation with rectangular pulse shaping and neglecting the effect of thermal noise, it is known that $\gamma$ can be expressed as [4]

$$
\gamma=\frac{3 S_{f}}{2 m}
$$

and the bit error probability assuming perfect coherent demodulation is given by

$$
P_{b}(\gamma)=\frac{1}{2} \operatorname{erfc}(\sqrt{\gamma})
$$


If we use a block error correcting code able to correct up to $t$ errors, we can evaluate the probability of having a correctable block, $P_{c}(\gamma)$, as:

$$
P_{c}(\gamma)=\sum_{n=0}^{t}\left(\begin{array}{l}
L \\
n
\end{array}\right) P_{b}(\gamma)^{n}\left(1-P_{b}(\gamma)\right)^{L-n} .
$$

Finally, the average block error probability BLER is given by

$$
\mathrm{BLER}=1-P_{c}(\gamma) .
$$

This analysis could be extended for other coding schemes (convolutional, turbo codes, etc.) by making use of the corresponding expression for $P_{c}(\gamma)$ of having a correct $L$-bit packet. However, these kind of expressions are usually code-dependent. Consequently, without loss of generality, we will just assume a block coding scheme for the example numerical results shown in Section III.

\section{B. All Users with Open-Loop Power Control}

We consider a group of $k+1$ users that apply open-loop power control. The mean received power, $\sigma$, is the same for all of them and we assume that they suffer from independent Rayleigh fadings. In these conditions, $\gamma$ can be expressed as

$$
\gamma=\frac{3 S_{f}}{2} \frac{\alpha_{0}^{2}}{\sum_{i=1}^{k} \alpha_{i}^{2}}=\frac{3 S_{f}}{2} \frac{X}{Y}
$$

where we have denoted the power received from the target user by $X=\alpha_{0}^{2}$, and the total interference level by $Y=\alpha_{1}^{2}+\cdots+$ $\alpha_{k}^{2}$. The pdf's of these random variables are

$$
\begin{gathered}
f_{X}(x)=\frac{1}{\sigma} e^{-(x / \sigma)} \quad x>0 \\
f_{Y}(y)=\frac{y^{k-1}}{\sigma^{k}(k-1) !} e^{-(y / \sigma)}, \quad y>0 .
\end{gathered}
$$

$\gamma$ is now a random variable, so it must be evaluated statistically in order to derive the block error probability. The probability distribution function of $\gamma$ is given by

$$
\begin{aligned}
F_{\gamma}(\gamma)= & P\left(\frac{3 S_{f}}{2} \frac{X}{Y} \leq \gamma\right) \\
= & \int_{0}^{\infty} e^{-(x / \sigma)} \int_{\left(3 S_{f} x / 2 \gamma\right)}^{\infty} \frac{1}{\sigma^{k+1}} \frac{y^{k-1}}{(k-1) !} \\
& \cdot e^{-(y / \sigma)} d y d x \\
= & 1-\left(\frac{2 \gamma}{3 S_{f}}+1\right)^{-k}, \quad \gamma>0 .
\end{aligned}
$$

Then, the pdf is immediately obtained by deriving the last expression with respect to variable $\gamma$,

$$
f_{\gamma}(\gamma)=\frac{2 k}{3 S_{f}}\left(\frac{2 \gamma}{3 S_{f}}+1\right)^{-k-1}, \quad \gamma>0
$$

Note that this pdf is quite similar to a Pareto distribution. Particularly, it is easily shown that the random variable $\beta=$ $\left(2 \gamma / 3 S_{f}\right)+1$ follows exactly a Pareto distribution of parameters $(1, k)$. As it occurs with this variable, its expected value equals infinity for $k=1$, while its variance is also infinity for $k=1$ and $k=2$. This variable $\beta$ has a physical meaning: It is the ratio between the total received power at the input (including both useful signal and interference) and the total interference power.

\section{Target User with Open-Loop Power Control and Mixed Interference}

We consider a user transmitting with open-loop power control and two groups of interferers: $k$ users also applying open-loop power control and $m$ users applying ideal closed-loop power control. Again, Rayleigh fading is independent between users. In this situation, the total signal-to-interference ratio $\gamma$ is expressed as

$$
\gamma=\frac{3 S_{f}}{2} \frac{\alpha_{0}^{2}}{m \sigma+\sum_{i=1}^{k} \alpha_{i}^{2}}=\frac{3 S_{f}}{2} \frac{X}{(m \sigma+Y)}
$$

where the random variables $X$ and $Y$ are the same as those defined in Section II-B, and assuming that $\sigma$ is the same for both types of users. This assumption arises from the fact that all users have the same quality requirements, that is, the same target received power.

The probability distribution function of $\gamma$ is given by:

$$
\begin{aligned}
F_{\gamma}(\gamma) & =P\left(\frac{3 S_{f}}{2} \frac{X}{Y+m \sigma} \leq \gamma\right) \\
& =P\left(\frac{3 S_{f}}{2 \gamma} X-m \sigma \leq Y\right) .
\end{aligned}
$$

Let us make the change $a=3 S_{f} / 2 \gamma$. In addition, we must distinguish between the intervals $x \geq m \sigma / a$ and $x<m \sigma / a$. Then, we can rewrite (11) as

$$
\begin{aligned}
P( & a X-m \sigma \leq Y) \\
= & \int_{0}^{(m / a) \sigma} e^{-(x / \sigma)} \int_{0}^{\infty} \frac{1}{\sigma^{k+1}} \frac{y^{k-1}}{(k-1) !} e^{-(y / \sigma)} d y d x \\
& +\int_{(m / a) \sigma}^{\infty} e^{-(x / \sigma)} \int_{a x-m \sigma}^{\infty} \frac{1}{\sigma^{k+1}} \frac{y^{k-1}}{(k-1) !} e^{-(y / \sigma)} d y d x \\
= & 1-e^{-(m / a)}+e^{-(m / a)}\left(1-\left(\frac{a}{1+a}\right)^{k}\right) \\
= & 1-\left(\frac{2 \gamma}{3 S_{f}}+1\right)^{-k} e^{-\left(2 m / 3 S_{f}\right) \gamma}, \quad \gamma>0
\end{aligned}
$$

The desired pdf is then given by

$$
\begin{aligned}
f_{\gamma}(\gamma)= & \frac{2}{3 S_{f}}\left(\frac{2 \gamma}{3 S_{f}}+1\right)^{-k}\left[k\left(\frac{2 \gamma}{3 S_{f}}+1\right)^{-1}+m\right] \\
& \cdot e^{-\left(2 m / 3 S_{f}\right) \gamma}, \quad \gamma>0 .
\end{aligned}
$$

Note that (9) is a particular case of this last expression when taking $m=0$.

\section{Target User with Closed-Loop Power Control and Mixed Interference}

A user transmitting with an ideal closed-loop power control is considered, and there are also two groups of interferers: $k$ users that apply an open-loop power control and $m$ users that apply an ideal closed-loop power control. Again, Rayleigh fading for 


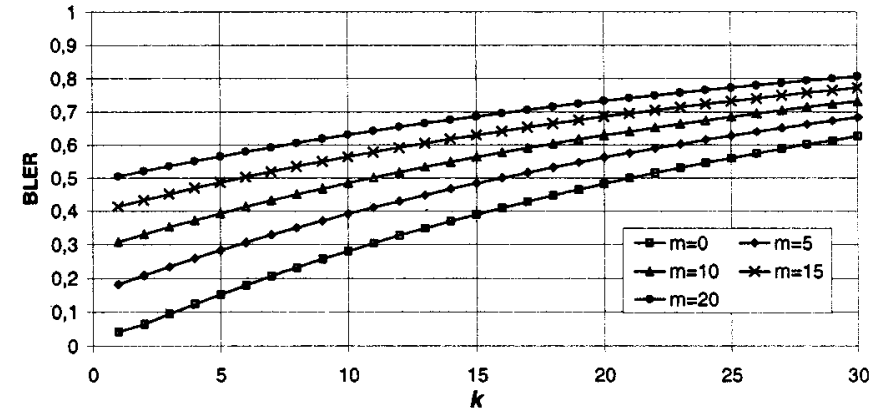

Fig. 1. Average block error probability for cases II.B $(m=0)$ and II.C.

interference is independent between users. Now, $\gamma$ can be expressed as

$$
\gamma=\frac{3 S_{f}}{2} \frac{\sigma}{m \sigma+\sum_{i=1}^{k} \alpha_{i}^{2}}=\frac{3 S_{f}}{2} \frac{\sigma}{(m \sigma+Y)}
$$

where $Y$ is defined as above.

The probability distribution function of $\gamma$ is

$$
\begin{aligned}
F_{\gamma}(\gamma)= & P\left(\frac{3 S_{f}}{2} \frac{\sigma}{Y+m \sigma} \leq \gamma\right) \\
= & \int_{\left(\left(3 S_{f} / 2 \gamma\right)-m\right) \sigma}^{\infty} \frac{y^{k-1}}{\sigma^{k}(k-1) !} e^{-(y / \sigma)} d y \\
= & e^{-\left(\left(3 S_{f} / 2 \gamma\right)-m\right)} \sum_{n=0}^{k-1} \frac{1}{(k-n-1) !} \\
& \cdot\left(\frac{3 S_{f}}{2 \gamma}-m\right)^{k-1-n}, \quad 0<\gamma<\frac{3 S_{f}}{2 m} ; k \geq 1 .
\end{aligned}
$$

The pdf of $\gamma$ is then

$$
\begin{aligned}
f_{\gamma}(\gamma)= & \frac{3 S_{f}}{2 \gamma^{2}(k-1) !}\left(\frac{3 S_{f}}{2 \gamma}-m\right)^{k-1} e^{-\left(\left(3 S_{f} / 2 \gamma\right)-m\right)}, \\
& 0<\gamma<\frac{3 S_{f}}{2 m}, \quad k \geq 1 .
\end{aligned}
$$

Note that $f_{\gamma}(\gamma)=0$ for $\gamma>3 S_{f} / 2 m$, that is, the signal-to-interference ratio is upper bounded by the value corresponding to the case in which all the users apply closed-loop power control.

\section{NUMERICAL RESULTS}

Figs. 1 and 2 show some numerical results for the BLER in cases II.B, II.C, and II.D, calculated as

$$
\mathrm{BLER}=1-\int_{0}^{\infty} P_{c}(\gamma) f_{\gamma}(\gamma) d \gamma
$$

where $P_{c}(\gamma)$ is given by (3) and $f_{\gamma}(\gamma)$ is defined in (9), (13), and (16), respectively.

The values presented were calculated with $S_{f}=64, L=$ 640 bits, and a block code with correcting capability $t=3$ er-

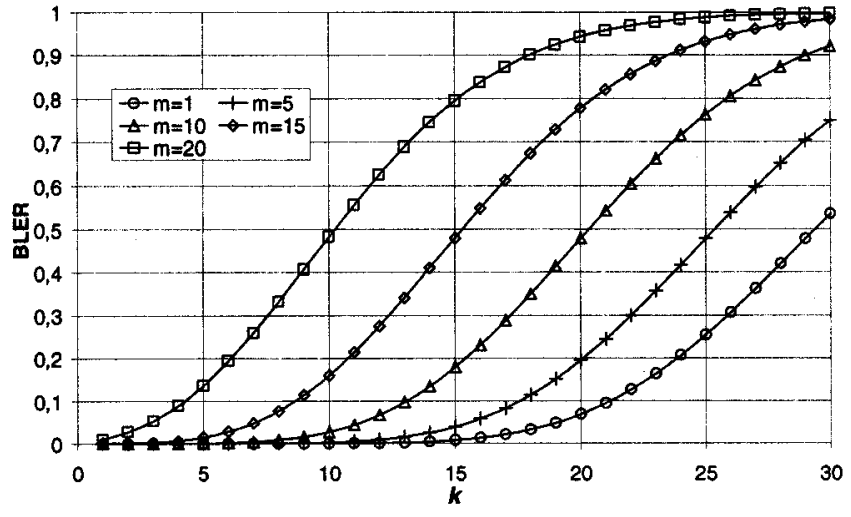

Fig. 2. Average block error probability for case II.D.

rors. Note that the BLER for case II.C increases less abruptly than for case II.D. Indeed, for low values of $k$ the BLER is lower for the closed-loop case. However, as $k$ increases, the BLER becomes better for the other power control technique. This behavior can be explained by the fact that, when the total interference level is high, it is better for a specific user to let its received power vary instead of maintaining it at a constant level. Then, when applying open-loop power control, the random nature of Rayleigh fading may allow some packets to arrive with a higher level than the average value, due to the constructive contribution of the multipath components of the transmitted signal, thus reducing the average block error probability.

\section{CONCLUSIONS}

We propose an analytical method to evaluate the average block error probability in a DS/CDMA packet radio transmission system where two different types of interferer sources coexist: Users applying either open-loop or closed-loop power control. The analytic expressions for the pdf's of the total signal-to-interference ratio for four different scenarios have been derived. The average block error probability is computed by making use of these pdf's. Finally, some numerical examples have been presented.

\section{REFERENCES}

[1] P. Díaz and R. Agustí, "The use of coding and diversity combining for mitigating fading effects in a DS/CDMA system," IEEE Trans. Veh. Technol., vol. 47, pp. 95-101, Feb. 1998.

[2] J. G. Proakis, Ed., Digital Communications. New York: McGraw-Hill, 1983.

[3] O. Sallent and R. Agustí, "A proposal for an adaptive S-ALOHA access system for a mobile CDMA environment," IEEE Trans. Veh. Technol., vol. 47, pp. 977-986, Aug. 1998.

[4] M. B. Pursley, "Performance evaluation for phase-coded spread spectrum multiple-access communication-Part I: System analysis," IEEE Trans. Commun., vol. COM-25, pp. 795-799, Aug. 1977.

[5] M. Simon and J. Omura, Spread Spectrum Communications. Rockville, MD: Computer Science Press, 1985, vol. I, p. 194. 